\title{
Strong-coupling expansion and effective hamiltonians
}

\author{
Frédéric Mila ${ }^{1}$ and Kai Phillip Schmidt ${ }^{2}$ \\ ${ }^{1}$ Institute of Theoretical Physics, Ecole Polytechnique Fédérale de Lausanne, \\ CH-1015 Lausanne, Switzerland; frederic.mila@epfl.ch \\ ${ }^{2}$ Lehrstuhl für Theoretische Physik I, TU Dortmund, D-44221 Dortmund, \\ Germany; schmidt@fkt.physik. uni-dortmund.de
}

When looking for analytical approaches to treat frustrated quantum magnets, it is often very useful to start from a limit where the ground state is highly degenerate. This chapter discusses several ways of deriving effective Hamiltonians around such limits, starting from standard degenerate perturbation theory and proceeding to modern approaches more appropriate for the derivation of high-order effective Hamiltonians, such as the perturbative continuous unitary transformations or contractor renormalization. In the course of this exposition, a number of examples taken from the recent literature are discussed, including frustrated ladders and other dimer-based Heisenberg models in a field, as well as the mapping between frustrated Ising models in a transverse field and quantum dimer models.

\subsection{Introduction}

As emphasized several times throughout this book, frustrated magnets often have a highly degenerate ground state in the classical limit. This is sometimes even taken as a definition of highly frustrated magnets. This degeneracy makes the semi-classical expansion in $1 / S$ effectively uncontrolled (if it does not already fail simply because of divergent quantum fluctuations), because usually one cannot perform and thus compare the expansions around all classical ground states. An infinite degeneracy is also often present in other limiting cases such as decoupled local units (such as triangles in the $S=1 / 2$ trimerized kagome lattice) or the Ising limit (for systems such as the antiferromagnetic Heisenberg model on the triangular or the kagome lattice). In such limits, which preserve the quantum nature of the problem, this degeneracy is not the end of the story, but rather the starting point of a systematic expansion, namely degenerate perturbation theory, which leads to an effective Hamiltonian. In the context of strongly correlated systems, this type of method usually goes by the name 'strong-coupling expansion,' because the starting point of 
the perturbative expansion is a Hamiltonian where only the interaction terms are kept, the kinetic terms being treated as the perturbation.

There are several ways to perform this expansion, or more generally to derive an effective Hamiltonian. There are in fact two types of effective Hamiltonian: those which act only in the degenerate subspace of a non-perturbed Hamiltonian, and those which act in the full Hilbert space but, through a canonical transformation, are rewritten as a series in terms of the ratio of two parameters. While the first type can always be written down explicitly, the second type can be derived in a simple way only provided that one can find a suitable generator. In the following, we will discuss both types of effective Hamiltonian, starting with the expansion in the degenerate subspace because this is more standard.

The derivation of an effective Hamiltonian is extremely useful in isolating the relevant degrees of freedom. However, the problem is usually not solved once the effective Hamiltonian has been derived. Indeed, the new Hamiltonian often poses a problem as difficult as the original one. The primary advantage of the effective Hamiltonian is that, because the relevant degrees of freedom have been selected, simple approximations to the problem defined by the effective Hamiltonian often give deep insight into the physics of the problem. We will illustrate this point with several examples.

This chapter is organized as follows. In Sec. II, we review briefly the degenerate perturbation theory approach to effective Hamiltonians, with a concise but self-contained discussion of the second-order result, and a description of the form the expansion takes at higher orders. In Sec. III, we discuss three examples taken from the field of frustrated magnetism where this approach has proven very useful: coupled dimers in a magnetic field, the Ising model in a transverse field, and the trimerized, spin- $1 / 2$ kagome antiferomagnet. In Sec. IV, we review more sophisticated approaches based on the same foundation: canonical transformations, continuous unitary transformations (CUTs), and the contractor renormalization group approach (CORE). Note that linked cluster expansions are discussed in another chapter of this book (that by Läuchli). We conclude in Sec. V with a discussion that includes a comparison of the various approaches.

\subsection{Strong-coupling expansion}

Let us consider a system described by a Hamiltonian

$$
H=H_{0}+V
$$

acting in a Hilbert space $\mathcal{H}$ such that the ground state of $H_{0}$ is degenerate. We denote by $\mathcal{H}_{0}$ the Hilbert space of the ground-state manifold. The goal is to find an effective Hamiltonian $H_{\text {eff }}$ acting in $\mathcal{H}_{0}$ such that

$$
H_{\text {eff }}|\phi\rangle=E|\phi\rangle \Rightarrow H|\psi\rangle=E|\psi\rangle, \quad|\phi\rangle \in \mathcal{H}_{0}, \quad|\psi\rangle \in \mathcal{H} .
$$




\subsubsection{Second-order perturbation theory}

Up to second order, the relation to be derived below is a standard result of quantum mechanics [1]. Denoting by $E_{0}$ the ground-state energy of $H_{0}$ and by $E_{m}$ the other eigenenergies, $H_{0}|m\rangle=E_{m}|m\rangle$ for $E_{m} \neq E_{0}$. Thus for two vectors $|\phi\rangle,\left|\phi^{\prime}\right\rangle \in \mathcal{H}_{0}$, and up to second order in $V$,

$$
\left\langle\phi\left|H_{\mathrm{eff}}\right| \phi^{\prime}\right\rangle=\left\langle\phi\left|H_{0}\right| \phi^{\prime}\right\rangle+\left\langle\phi|V| \phi^{\prime}\right\rangle+\sum_{|m\rangle \notin \mathcal{H}_{0}} \frac{\langle\phi|V| m\rangle\left\langle m|V| \phi^{\prime}\right\rangle}{E_{0}-E_{m}} .
$$

This result can be reformulated as an operator identity [2]. If one denotes by $P$ the projector on $\mathcal{H}_{0}$, and defines $Q=1-P$, then to second order in $V$

$$
P H_{\mathrm{eff}} P=P H_{0} P+P V P+P V Q \frac{1}{E_{0}-Q H_{0} Q} Q V P .
$$

Proof: suppose $H|\psi\rangle=E|\psi\rangle$. Because $P+Q=1$, this can be written as

$$
(P+Q) H(P+Q)|\psi\rangle=E|\psi\rangle .
$$

Projecting onto $\mathcal{H}_{0}$ and $\mathcal{H}-\mathcal{H}_{0}$ gives

$$
\begin{aligned}
& P H P|\psi\rangle+P H Q|\psi\rangle=E P|\psi\rangle, \\
& Q H P|\psi\rangle+Q H Q|\psi\rangle=E Q|\psi\rangle,
\end{aligned}
$$

whence

$$
\begin{gathered}
(2) \Rightarrow Q|\psi\rangle=(E-Q H Q)^{-1} Q H P|\psi\rangle \\
(1) \Rightarrow P H P|\psi\rangle+P H Q \frac{1}{E-Q H Q} Q H P|\psi\rangle=E P|\psi\rangle .
\end{gathered}
$$

Expansion of $(E-Q H Q)^{-1}$ using $(A-B)^{-1}=A^{-1} \sum_{n=0}^{\infty}\left(B A^{-1}\right)^{n}$, with $A=E_{0}-Q H_{0} Q$ and $B=Q V Q-E+E_{0}$, leads to

$$
\begin{aligned}
P H_{\mathrm{eff}} P= & P H_{0} P+P V P+P V Q\left(E_{0}-Q H_{0} Q\right)^{-1} \\
& \times \sum_{n=0}^{\infty}\left(\left(Q V Q-E+E_{0}\right)\left(E_{0}-Q H_{0} Q\right)^{-1}\right)^{n} Q V P .
\end{aligned}
$$

The expansion is then truncated at $n=0$. QED.

\subsubsection{High-order perturbation theory}

This form of the expansion is not well suited to the derivation of higherorder expansions, because beyond second order it contains explicitly the exact eigenenergy $E$. An expansion only in terms of the unperturbed eigenenergies can nevertheless be derived. The first systematic method for this dates to the 
work of Kato [3]. Here we follow the formulation of Takahashi [4], in which the expansion takes the form

$$
\begin{gathered}
P H_{\mathrm{eff}} P=\Gamma^{\dagger} H \Gamma \\
\Gamma=\bar{P} P(P \bar{P} P)^{-1 / 2}, \\
(P \bar{P} P)^{-1 / 2}=P+\sum_{n=1}^{\infty} \frac{(2 n-1) ! !}{(2 n) ! !}[P(P-\bar{P}) P]^{n}, \\
\bar{P}=P-\sum_{n=1}^{\infty} \sum_{k_{1}+\ldots+k_{n+1}=n, k_{i} \geq 0} S^{k_{1}} V S^{k_{2}} V \ldots V S^{k_{n+1}}, \\
S^{0}=-P, \quad S^{k}=\left(\frac{Q}{E_{0}-Q H_{0} Q}\right)^{k} .
\end{gathered}
$$

The true eigenstates $\psi$ are related to the eigenstates $\phi$ of $H_{\text {eff }}$ by

$$
|\phi\rangle=\Gamma|\psi\rangle,
$$

and likewise the observables transform according to

$$
O \rightarrow \Gamma^{\dagger} O \Gamma
$$

Thus the $n$th order term of $H_{\text {eff }}$ has the form

$$
H_{\mathrm{eff}}^{(n)}=\sum_{k_{1}+\ldots+k_{n-1}=n-1, k_{i} \geq 0} f\left(k_{1}, k_{2}, \ldots, k_{n-1}\right) V S^{k_{1}} V S^{k_{2}} V \ldots S^{k_{n-1}} V,
$$

where the coefficients $f\left(k_{1}, \ldots, k_{n-1}\right)$ are deduced by appropriate bookkeeping from the previous expansions. The number of terms in such a strong-coupling expansion grows exponentially with $n$. In practice, beyond the fourth order it can generally be carried out only with the help of a computer. An alternative formulation based on continuous unitary transformations, which is simpler when applicable, will be discussed in the next section. In the remainder of this section, we discuss a number of selected examples where low-order degenerate perturbation theory provides considerable additional insight into the problem.

\subsubsection{Examples}

In the field of quantum magnetism, the best known example is the derivation of the Heisenberg model starting from the half-filled Hubbard model. The Hubbard model is defined by

$$
H=V+H_{0}=-t \sum_{\langle i, j\rangle, \sigma}\left(c_{i \sigma}^{\dagger} c_{j \sigma}+\text { h.c. }\right)+U \sum_{i} n_{i \uparrow} n_{i \downarrow} .
$$




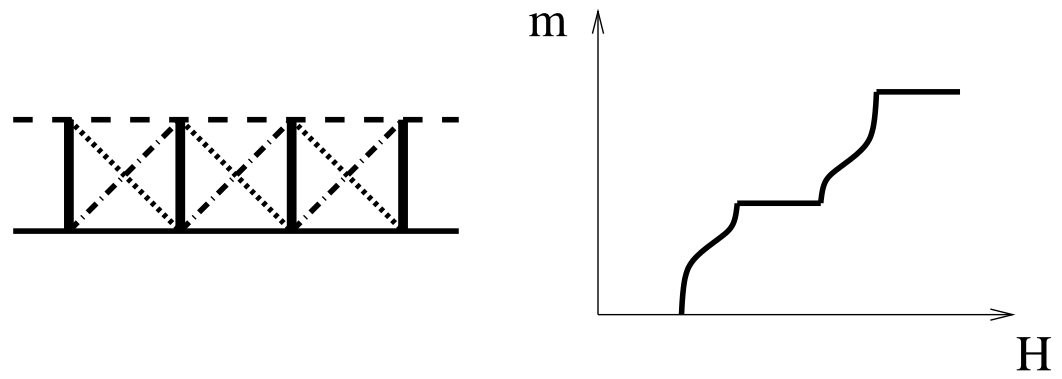

Fig. 1.1. Left: schematic representation of the spin ladder of Eq. 1.2. The couplings entering Eq. 1.2 are denoted by the different line types: thick solid $\left(J_{\perp}\right)$, thin solid $\left(J_{11}\right)$, dashed $\left(J_{22}\right)$, dot-dashed $\left(J_{12}\right)$ and dotted $\left(J_{21}\right)$. Right: magnetization curve of a frustrated spin ladder with a 1/2-magnetization plateau.

At half-filling, the ground state of the interaction term $H_{0}$ is $2^{N}$-fold degenerate, where $N$ is the number of sites. Treating the kinetic term $V$ as a perturbation leads at second order (up to a constant) to the effective Hamiltonian

$$
H_{\mathrm{eff}}=J \sum_{\langle i, j\rangle} \boldsymbol{S}_{i} \cdot \boldsymbol{S}_{j}
$$

with $J=4 t^{2} / U[5]$. As noted in the introduction, this is a case where the effective Hamiltonian is itself very difficult to solve, which is indeed true for the Heisenberg model on most lattices. As we shall see in the following, it is often useful to go one step further, and to derive a further effective Hamiltonian starting from one physically relevant limit.

\section{Example 1: frustrated spin-1/2 ladder in a magnetic field}

We consider the Heisenberg model for a frustrated spin-1/2 ladder in a magnetic field defined by the Hamiltonian

$$
H=\sum_{n} J_{\perp} \boldsymbol{S}_{1 n} \cdot \boldsymbol{S}_{2 n}-B \sum_{n}\left(S_{1 n}^{z}+S_{2 n}^{z}\right)+\sum_{n} \sum_{i, j=1,2} J_{i j} \boldsymbol{S}_{i n} \cdot \boldsymbol{S}_{j n+1} .
$$

In the spin operators $\boldsymbol{S}_{i n}$, the index $i$ refers to the leg and the index $n$ to the rung (Fig. 1.1). The goal is to derive an effective Hamiltonian that describes the magnetization process in the limit $J_{\perp} \gg J_{i j}[6,7]$.

The starting point of the perturbative expansion is the Hamiltonian of isolated dimers at the critical field $B_{c}=J_{\perp}$ where, for one dimer, the triplet polarized in the field direction crosses the singlet,

$$
H_{0}=\sum_{n} J_{\perp} \boldsymbol{S}_{1 n} \cdot \boldsymbol{S}_{2 n}-B_{c} \sum_{n}\left(S_{1 n}^{z}+S_{2 n}^{z}\right) .
$$


The ground state of this Hamiltonian is $2^{N}$-fold degenerate, where $N$ is the number of ladder rungs, and the full Hamiltonian can be treated within degenerate perturbation theory, the perturbation being given by

$$
V=\sum_{\langle n m\rangle} \sum_{i, j=1,2} J_{i j} \boldsymbol{S}_{i n} \cdot \boldsymbol{S}_{j m}-\left(B-B_{c}\right) \sum_{n}\left(S_{1 n}^{z}+S_{2 n}^{z}\right) .
$$

The sum over $\langle n m\rangle$ refers to the nearest-neighbor rungs. A perturbative expansion can be performed under the condition that the matrix elements of $V$ are small compared to the excited states of $H_{0}$, i.e. as long as $J_{i j},\left(B-B_{c}\right) \ll$ $J_{\perp}$. The condition on $B-B_{c}$ might suggest that such a calculation cannot give access to the full magnetization curve, but in fact the magnetization is rigorously equal to zero or to the full saturation value outside a window whose width is of order $J_{i j}$. Thus this type of perturbation theory can indeed give the full magnetization curve.

To write down the effective Hamiltonian, one needs a description of the Hilbert space. Because there are two states per rung, one simple choice is to introduce the Pauli matrices $\boldsymbol{\sigma}_{n}$ at each rung such that the singlet corresponds to $\left|\sigma_{n}^{z}=-1 / 2\right\rangle$ and the triplet polarized along the field to $\left|\sigma_{n}^{z}=1 / 2\right\rangle$. In this basis, and up to first order in $V$, the effective Hamiltonian is given by

$$
H_{\mathrm{eff}}=J^{x y} \sum_{n}\left(\sigma_{n}^{x} \sigma_{n+1}^{x}+\sigma_{n}^{y} \sigma_{n+1}^{y}\right)+J^{z} \sum_{n} \sigma_{n}^{z} \sigma_{n+1}^{z}-B_{\mathrm{eff}} \sum_{n}\left(\sigma_{n}^{z}\right)+C,
$$

with

$$
\begin{aligned}
J^{x y} & =\frac{1}{2}\left(J_{11}+J_{22}-J_{12}-J_{21}\right), \\
J^{z} & =\frac{1}{4}\left(J_{11}+J_{22}+J_{12}+J_{21}\right), \\
B_{\mathrm{eff}} & =B-B_{c}-\frac{1}{4}\left(J_{11}+J_{22}+J_{12}+J_{21}\right), \\
C & =\frac{1}{8}\left(J_{11}+J_{22}+J_{12}+J_{21}\right)-\left(B-B_{c}\right) .
\end{aligned}
$$

The effective Hamiltonian is identical to that of an XXZ chain in a field, which is a major step forward with respect to the original problem: this model has been investigated at length using the Bethe ansatz [8] and by field-theory methods $[9,10]$, and much is known about its low-energy properties. In particular, there is a quantum phase transition in zero field at $J^{z}=J^{x y}$ between a gapless phase at small $J^{z}$ and a gapped phase at large $J^{z}$.

To understand the physics of this phase transition for the original problem, it is expedient to perform a Jordan-Wigner transformation [11] of the effective model. In this language, the Hilbert space is that of spinless fermions on a chain, and the elementary operators are fermion creation $\left(c_{i}^{\dagger}\right)$, annihilation $\left(c_{i}\right)$ and density $\left(n_{i}=c_{i}^{\dagger} c_{i}\right)$ operators at site $i$. An empty site corresponds to a rung singlet, an occupied one to a rung triplet, and the effective Hamiltonian becomes 


$$
H_{\mathrm{eff}}=-t \sum_{i}\left(c_{i}^{\dagger} c_{i+1}+c_{i+1}^{\dagger} c_{i}\right)+v \sum_{i} n_{i} n_{i+1}-\mu \sum_{n} n_{i},
$$

with $t=J^{x y} / 2, v=J^{z}$, and $\mu=B_{\text {eff }}+J^{z}$. In this model, the gapped phase of the ladder is a half-filled insulating phase of the fermionic chain, while the gapless one is a metallic phase (a Luttinger liquid in this one-dimensional (1D) system). Thus the density as a function of the chemical potential has a plateau, the width of which is equal to the gap of the insulating phase. In the original model, this implies that the magnetization can have a plateau for certain parameters, a result which has been confirmed by density-matrix renormalization-group (DMRG) calculations [12]. The physics of the plateau state is discussed elsewhere in this volume (the chapter by Takigawa and Mila). For the purposes of the present chapter, we note only how powerful the effective-Hamiltonian method can be: a very simple, first-order calculation can basically solve the problem by mapping it onto another non-trivial but well-understood one.

\section{Example 2: expansion around the Ising limit}

In the previous example, as in the case of the Hubbard model, the unperturbed Hamiltonian is a sum of local terms, and the macroscopic ground-state degeneracy is given simply from the ground-state degeneracy of each term. This is not the only case where the effective-Hamiltonian approach is useful. Another important example is the ground state of the antiferromagnetic Ising model, which is degenerate on non-bipartite lattices such as the triangular and kagome geometries. Again this can be the starting point of a degenerate perturbation theory towards the Heisenberg model on the same lattice if the transverse exchange is treated as a perturbation. In the same spirit, degenerate perturbation theory can be used to treat the effect of a transverse magnetic field applied to a frustrated Ising model. In this section we concentrate on the Ising case, which is of direct relevance to Quantum Dimer Models (QDMs, discussed in the chapter by Moessner and Raman).

Consider the fully frustrated Ising model in a transverse field on the honeycomb lattice, defined by the Hamiltonian

$$
H=H_{0}+V=-J \sum_{\langle i, j\rangle} M_{i j} \sigma_{i}^{z} \sigma_{j}^{z}-\Gamma \sum_{i} \sigma_{i}^{x} .
$$

In this expression, the parameters $M_{i j}= \pm 1$ are chosen in such a way that their product around each hexagonal plaquette is equal to -1 . All models in this class are then equivalent up to a gauge transformation, $\sigma_{i}^{z} \rightarrow \epsilon_{i} \sigma_{i}^{z}$, $M_{i j} \rightarrow \epsilon_{i} \epsilon_{j} M_{i j}$, with $\epsilon_{i}= \pm 1$. One possible choice is shown in Fig. 1.2. For this particular choice, the two ferromagnetic states, with all spins either up or down, are ground states of $H_{0}$. Indeed, they satisfy five bonds out of every six, and it is clearly impossible to do better for a case with one antiferromagnetic coupling out of six. From a ferromagnetic ground state, one 

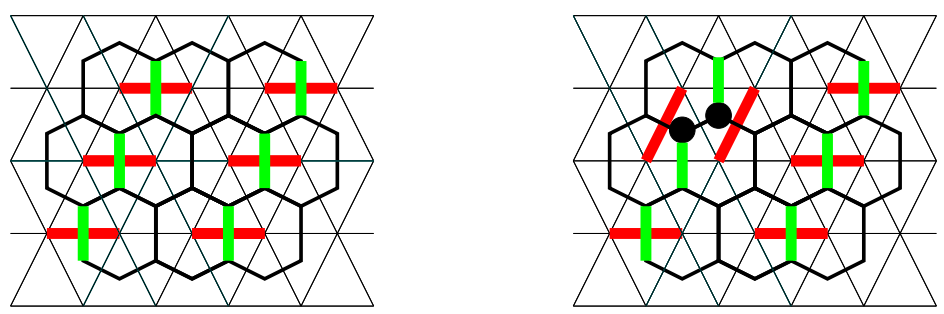

Fig. 1.2. Left: representation of the model of Eq. 1.11. The thick vertical lines on the honeycomb lattice correspond to $M_{i j}=-1$, all others to $M_{i j}=1$. The thick horizontal lines on the triangular lattice correspond to dimers in the ferromagnetic ground state. Right: dimer covering obtained from the ferromagnetic ground state after flipping the two spins shown as black dots.

may then construct more ground states, and it is easy to verify that flipping the spins at the ends of a satisfied bond that connects two unsatisfied bonds leads to another ground state (right panel, Fig. 1.2).

The structure of the Hilbert space is best understood by considering the dual lattice, which is the triangular lattice of sites at the centers of the hexagonal plaquettes, and to draw a line between two neighboring sites of the dual lattice if the bond (of the original lattice) which it crosses is unsatisfied. If one imposes the constraint that each site be connected to exactly one of its neighbors, this set of lines defines a dimer covering of the triangular lattice, and there is a one-to-one correspondence between these dimer coverings and the ground states of $H_{0}$ which have a given spin orientation for each given site. Because there are two possible spin orientations, the degenerate manifold of $H_{0}$ is twice as large as the Hilbert space of dimer coverings, the two configurations leading to the same dimer covering being related by a global flip of all spins.

For simplicity, we focus on the problem defined by the Ising Hamiltonian acting in the Hilbert space where two configurations related by a global spinflip are identified. Then the degenerate subspace can be described by the set of dimer coverings, and the effective Hamiltonian obtained by degenerate perturbation theory takes the form of a QDM. To deduce the form of this QDM, we first note that the effective Hamiltonian vanishes to first order in the transverse field. Indeed, flipping a single spin changes the nature of the three bonds connected to it, which increases the number of unsatisfied bonds by one or by three, depending on whether or not one of the bonds connected to this site was unsatisfied. To second order, the situation is still more complex unless the two sites are nearest neighbors, in which case the process does not result in a state outside the ground-state manifold provided the two sites are on a satisfied bond connected to two unsatisfied ones. The resulting process leads to a dimer flip around a square plaquette of the dual lattice, and the effective Hamiltonian is a special case of the Rokhsar-Kivelson model, defined by the Hamiltonian 


$$
\begin{aligned}
& \mathcal{H}=-t \sum_{r}(\mid \bullet \bullet\langle\bullet \bullet|+\text { H.c. })
\end{aligned}
$$

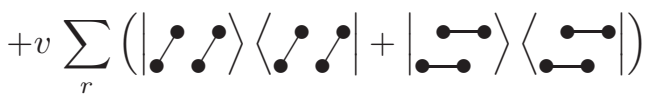

with $t=\Gamma^{2} / J$ and $v=0$.

In this example, it is the effective model which is of direct interest in the context of frustrated magnets (as discussed in the chapter by Moessner and Raman). The connection with the Ising model turns out to be helpful in identifying a possible phase transition, as first noted in Ref. [13], and led more recently to an analytical description of the fractional excitations which exist in the Resonating-Valence-Bond phase of the QDM on the square lattice [14].

As mentioned at the beginning of this section, an expansion around the Ising limit can also be performed for the XXZ version of the Heisenberg model, using the ratio $J^{x y} / J^{z}$ as a small parameter. This approach has been used for the 1/3-plateau of the spin-1/2 kagome antiferromagnet [15], for the halfmagnetization plateau phase of the spin-1/2 pyrochlore Heisenberg antiferromagnet [16], and for the 1/3-plateau phase of molecular analogs of the spin $1 / 2$ kagome antiferromagnet [17].

\section{Example 3: weakly coupled triangles}

Frustration naturally appears in antiferromagnets in which the exchange paths create loops of odd length, the simplest of these loops being the triangle. There are several types of lattice geometry which can be considered as coupled triangles, and for which a perturbation theory starting from non-interacting triangles has proven to be useful. The effective model takes a special form due to the pecularity of the ground-state manifold of a triangle, which is four-fold degenerate. This is easily seen by rewriting the Hamiltonian in terms of the total spin of the triangle, $\boldsymbol{S}_{\text {tot }}=\boldsymbol{S}_{1}+\boldsymbol{S}_{2}+\boldsymbol{S}_{3}$, to obtain

$$
H=J\left(\boldsymbol{S}_{1} \cdot \boldsymbol{S}_{2}+\boldsymbol{S}_{2} \cdot \boldsymbol{S}_{3}+\boldsymbol{S}_{3} \cdot \boldsymbol{S}_{1}\right)=\frac{J}{2}\left[\left(\boldsymbol{S}_{\mathrm{tot}}\right)^{2}-\frac{9}{4}\right],
$$

a result emerging because it is possible to construct two doublets, i.e. four states, using three spin- $1 / 2$ entities. A convenient basis is provided by the simultaneous eigenstates of the scalar chirality, $\boldsymbol{S}_{\mathbf{1}} \cdot\left(\boldsymbol{S}_{\mathbf{2}} \times \boldsymbol{S}_{\mathbf{3}}\right)$, and of the projection $S_{\text {tot }}^{z}$ of the total spin,

$$
\begin{aligned}
& |R, \sigma\rangle=\left(|-\sigma \sigma \sigma\rangle+\omega|\sigma-\sigma \sigma\rangle+\omega^{2}|\sigma \sigma-\sigma\rangle\right) / \sqrt{3} \\
& |L, \sigma\rangle=\left(|-\sigma \sigma \sigma\rangle+\omega^{2}|\sigma-\sigma \sigma\rangle+\omega|\sigma \sigma-\sigma\rangle\right) / \sqrt{3}
\end{aligned}
$$

where $\omega=\exp (2 i \pi / 3), \sigma= \pm 1 / 2$ refers to $S_{\text {tot }}^{z}$, and $L$ and $R$ represent leftand right-handed chirality.

In a system of weakly coupled triangles, treating the inter-triangle coupling as a perturbation leads to an effective Hamiltonian which acts in a Hilbert 
space of dimension $4^{N_{t}}$, where $N_{t}$ is the number of triangles. For a given triangle $i$, it is convenient to introduce a spin- $1 / 2$ operator $\boldsymbol{\sigma}_{i}$, acting on the total spin, and a Pauli-matrix vector $\boldsymbol{\tau}_{i}$ acting in chirality space. To first order in the inter-triangle couplings, provided these couplings are $\mathrm{SU}(2)$-invariant, the effective Hamiltonian then takes the general form

$$
H_{\mathrm{eff}}=J_{\mathrm{eff}} \sum_{i, j}^{\prime} \boldsymbol{\sigma}_{i} \cdot \boldsymbol{\sigma}_{j} H_{i j}^{\tau}
$$

where the sum is over the lattice of sites representing the arrangement of the triangles, $J_{\text {eff }}$ is linear in the inter-triangle couplings, and the operator $H_{i j}^{\tau}$ depends on which sites of the triangles $i$ and $j$ are coupled by the inter-triangle interaction.

In the case of a three-leg 'spin tube,' the effective model takes the explicit form $[18]$

$$
H_{\mathrm{eff}}=\frac{J^{\prime}}{3} \sum_{\langle i j\rangle}^{\prime} \boldsymbol{\sigma}_{i} \cdot \boldsymbol{\sigma}_{j}\left[1+2\left(\tau_{i}^{x} \tau_{i+1}^{x}+\tau_{i}^{x} \tau_{i+1}^{x}\right)\right],
$$

where $J^{\prime}$ is the rung coupling of the real model. For the effective model, field-theory arguments based on bosonization show that the spectrum must be gapped in all sectors, a non-trivial prediction to be contrasted with the gapless spectrum of the three-leg ladder [18].

Another example where this type of effective Hamiltonian has provided additional valuable insight is on the kagome lattice. Considering the trimerized version of this model, where the exchange constant within up-pointing triangles is taken to be $J$ and in down-pointing triangles $J^{\prime}$, the effective Hamiltonian in the limit $J^{\prime} \ll J$ can be expressed as $[19,20]$

$$
H_{\mathrm{eff}}=\frac{J^{\prime}}{9} \sum_{\langle i j\rangle}^{\prime} \boldsymbol{\sigma}_{i} \cdot \boldsymbol{\sigma}_{j}\left(1-4 \boldsymbol{e}_{i j} \cdot \boldsymbol{\tau}_{i}\right)\left(1-4 \boldsymbol{e}_{i j} \cdot \boldsymbol{\tau}_{j}\right),
$$

where the vectors $\boldsymbol{e}_{i j}$ are taken from among $\boldsymbol{e}_{1}=(1,0), \boldsymbol{e}_{2}=(-1 / 2,-\sqrt{3} / 2)$, and $\boldsymbol{e}_{3}=(-1 / 2, \sqrt{3} / 2)$, the choice depending on the labelling of the spins inside each triangle [21]. For this effective Hamiltonian, a mean-field decoupling of spin and chirality leads to a highly degenerate ground state. Each ground state can be associated with a dimer covering of the triangular lattice of up- (or down-)pointing triangles, and the number of states accordingly grows as $1.5351^{N_{t}}$ in terms of the number of triangles, or as $1.1536^{N}$ in terms of the number of spins [20]. This compares well with the number of low-lying singlets observed in numerical simulations of the spin-1/2 kagome antiferromagnet [22].

This effective Hamiltonian is also a very useful starting point to discuss the physics in a magnetic field. Antiferromagnets composed of weakly coupled triangles exhibit a plateau at magnetization $1 / 3$, in which all triangles have a total spin equal to $1 / 2$ (in a $S=1 / 2$ system) and oriented in the field 
direction. Inside the plateau, $\boldsymbol{\sigma}_{i} \cdot \boldsymbol{\sigma}_{j}=1 / 4$ is a constant, and the effective Hamiltonian is a pure chirality model. This plateau has been investigated for the kagome lattice in Ref. [23].

Finally, similar ideas based on weakly coupled tetrahedra have provided equally helpful insight into the properties of the spin- $1 / 2$ Heisenberg model on the pyrochlore lattice and in related systems $[24,25]$.

\subsection{Alternative approaches yielding effective Hamiltonians}

There are several ways of deriving effective Hamiltonians based on techniques other than direct strong-coupling expansions. The aim of this section is to provide a review of the physics underlying these approaches, with appropriate references for further reading concerning their more detailed implementations.

\subsubsection{Canonical transformation}

The canonical transformation of a Hamiltonian is defined by $H \rightarrow \tilde{H}=$ $e^{\eta} H e^{-\eta}$, where $\eta$ is antihermitian, so that $e^{\eta}$ is unitary. If $|\psi\rangle$ is an eigenstate of $H$, then $|\tilde{\psi}\rangle=e^{\eta}|\psi\rangle$ is an eigenstate of $\tilde{H}$ with the same eigenvalue. If the operators are transformed simultaneously according to $A \rightarrow \tilde{A}=e^{\eta} A e^{-\eta}$, then $\langle\tilde{\psi}|\tilde{A}| \tilde{\psi}\rangle=\langle\psi|A| \psi\rangle$.

The foundation for using a canonical transformation to derive an effective Hamiltonian is the identity

$$
\begin{aligned}
e^{\eta} H e^{-\eta} & =H+[\eta, H]+\frac{1}{2 !}[\eta,[\eta, H]]+\ldots \\
& =H+\sum_{n=1}^{\infty} \frac{1}{n !}[\eta,[\eta, \ldots[\eta, H] \ldots]],
\end{aligned}
$$

which is simply the Taylor expansion of $H(\lambda)=e^{\lambda \eta} H e^{-\lambda \eta}$ for $\lambda=1$.

Considering the case where $H=H_{0}+\lambda V$, if one can find an operator $\eta$ such that $\left[\eta, H_{0}\right]=-V$, then using $\lambda \eta$ as a generator leads to

$$
\tilde{H}=H_{0}+\sum_{n=1}^{\infty} \frac{n \lambda^{n+1}}{(n+1) !}[\eta,[\eta, \ldots[\eta, V] \ldots]] .
$$

This operator is a series in powers of $\lambda$, and hence of the perturbation $V$. While its structure is reminiscent of the results of high-order perturbation theory, there is an important difference: $\tilde{H}$ acts in the full Hilbert space of $H$, whereas $H_{\text {eff }}$ acts in the ground-state manifold of $H_{0}$. Depending on the problem, this may or may not be an advantage. If one is interested in highenergy states which may be detected in a particular experiment, the canonicaltransformation approach has distinct advantages, because it gives all of the 
eigenstates up to a certain order, and not only those which have evolved from the ground-state manifold of $H_{0}$ under the perturbation. By contrast, if the goal is to reduce the Hilbert space to study the low-energy sector using, for ex-

ample, exact-diagonalization calculations on finite clusters, then a degenerate perturbation theory is sufficient.

\subsubsection{CUT (Continuous Unitary Transformation)}

The canonical transformation introduced in the previous section is by no means the only possibility for obtaining an effective model by a unitary transformation. In fact there are many ways to do this, even in low orders of a perturbative approach, and it is therefore an obvious question to ask whether an optimal choice of transformation exists.

This question led both Wegner [30] and Głazek and Wilson [31,32] to introduce independently of each other the concept of continuous unitary transformations (CUTs) [33]. In contrast to the one-step transformation discussed in the last section, here the unitary transformation is constructed as an infinite product of infinitesimal transformations. Although measurable (on-shell) quantities, such as energies, have to be the same independent of which kind of transformation has been chosen, off-shell quantities such as effective interactions can differ strongly. This has been demonstrated in a quite impressive manner for the case of the Fröhlich Hamiltonian, which describes conventional superconductivity mediated by the electron-phonon interaction [34]. Here the effective electron-electron interaction at second order in the electron-phonon coupling shows divergences for the case of a one-step transformation (previous section), whereas in the continuous treatment the attractive interaction is smooth.

Another respect in which the approaches differ is that the one-step canonical transformation can be applied practically only at low orders in the perturbation. However, there are physical situations where one is interested in the quantitative determination of an effective Hamiltonian for a given parameter set in the original model. One example is the case of strongly frustrated networks of coupled dimers, as in the Shastry-Sutherland model, where processes relevant to the magnetization of the system appear only at high orders.

In the method of CUTs, a continuous parameter $l$ is introduced such that $l=0$ refers to the initial system $H$ and $l=\infty$ corresponds to the final effective system, which should correspond to a simplified physical picture. The transformation can be constructed such that processes at higher energies are treated before those at lower energies. This renormalizing property is similar to Wilson's renormalization-group approach [35].

Let $U$ be the unitary transformation which diagonalizes the Hamiltonian $H$ and let $H(l)=U^{\dagger}(l) H U(l)$. This unitary transformation is then equivalent to performing an infinite sequence of unitary transformations, $e^{-\eta(l) d l}$, with the antihermitian generators 


$$
\eta(l)=-U^{\dagger}(l) \partial_{l} U(l)
$$

Taking the derivative with respect to $l$ results in the "flow equation"

$$
\partial_{l} H(l)=[\eta(l), H(l)]
$$

which defines the change of the Hamiltonian during the flow. Note that Eq. 1.18 represents an infinite hierarchy of coupled differential equations, because for the general case an increasing number of terms is generated on the right-hand side at each order. In practice, one has therefore to perform a truncation (below).

The properties of the effective Hamiltonian depend strongly on the choice of the generator. There are in essence two different modern variants. The first one uses the generator introduced originally by Wegner [30], which aims to eliminate interaction matrix elements with the goal of obtaining an energydiagonal effective Hamiltonian. This approach has been applied successfully to a large class of problems, with special attention being given to determining the ground state of interacting quantum many-body problems [30,34,36]. The second variant is the quasiparticle-conserving CUT which, as its name suggests, maps the Hamiltonian $H_{0}$ to an effective Hamiltonian conserving the number of quasiparticles [37-40]. This approach can be used either to study the excitations of an already known quantum ground state [33, 40-44], one application for which has been to bound states, or, in analogy to the previous case, to derive effective low-energy models [45-49].

Returning to the point mentioned above, in all practical calculations it is necessary to truncate the flow equation (1.18). For this there are two options: i) cutting the hierarchy at one level and solving the equations numerically (self-similar CUTs) or ii) using a series-expansion ansatz for $\eta$ and $H$, and solving the flow equations perturbatively to high order (perturbative CUTs).

Here we focus only on presenting one illustrative example, for which we choose the perturbative version of quasiparticle-conserving CUTs $[37,40,50$, 51]. If the problem at hand meets the two conditions:

1. the unperturbed part has an equidistant spectrum bounded from below,

2. there is an integer number $N>0$ such that the perturbing part can be separated as $\sum_{n=-N}^{N} T_{n}$, where $T_{n}$ increments (or if $n<0$ decrements) the number of particles by $n$,

then the CUT in its quasiparticle-conserving form can be solved to high order in the perturbation and the effective Hamiltonian is given by the general form $[40]$

$$
H_{\mathrm{eff}}(x)=Q+\sum_{k=1}^{\infty} x^{k} \sum_{\substack{|\underline{m}|=k \\ M(\underline{m})=0}} C(\underline{m}) T(\underline{m}),
$$

where $Q$ is the unperturbed part of the Hamiltonian, $x$ an expansion parameter, $\underline{m}=\left(m_{1}, m_{2}, \ldots, m_{k}\right)$, and $M(\underline{m})=\sum_{i=1}^{k} m_{i}=0$ reflects the conservation of the number of particles. The action of $H_{\text {eff }}$ can be viewed as a 
weighted sum of particle-number-conserving virtual excitation processes, each of which is encoded in a monomial $T(\underline{m})=T_{m_{1}} T_{m_{2}} \ldots T_{m_{k}}$. The coefficients $C(\underline{m})$ are rational numbers which can be calculated (to high order in the perturbation) exactly as the ratio of two integers. It should be emphasized that the effective Hamiltonian $H_{\text {eff }}$, which has known coefficients $C(\underline{m})$, can be used straightforwardly in all perturbative problems that meet the above conditions.

We illustrate the method first for an unfrustrated spin ladder in a magnetic field, including from Eq. (1.2) only the magnetic exchange $J_{\perp}$ on the rungs, the magnetic field $B$, and the unfrustrated exchange along the legs of the ladder, setting $J_{11}=J_{22} \equiv J_{\|}$. Rewriting the Hamiltonian in terms of rung triplet operators $t_{\alpha}$, with $\alpha=\{x, y, z\}$ [52], we obtain

$$
H=J_{\perp} Q+\frac{J_{\|}}{2}\left[T_{0}+T_{+2}+T_{-2}\right]+H_{B},
$$

where

$$
\begin{aligned}
Q & =\sum_{i, \alpha} t_{\alpha, i}^{\dagger} t_{\alpha, i}, \\
T_{0} & =\sum_{i, \alpha} t_{\alpha, i}^{\dagger} t_{\alpha, i+1}+\sum_{i, \alpha, \beta}\left[t_{\alpha, i}^{\dagger} t_{\beta, i+1}^{\dagger} t_{\beta, i} t_{\alpha, i+1}-t_{\alpha, i}^{\dagger} t_{\alpha, i+1}^{\dagger} t_{\beta, i} t_{\beta, i+1}\right], \\
T_{+2} & =\sum_{i, \alpha} t_{\alpha, i}^{\dagger} t_{\alpha, i+1}^{\dagger}, \\
T_{-2} & =\sum_{i, \alpha} t_{\alpha, i} t_{\alpha, i+1}=T_{+2}^{\dagger} .
\end{aligned}
$$

The operator $Q$ counts the total number of triplet excitations, while the operators $T_{n}$ change the triplet number by $n$, and $H_{B}$ denotes the magnetic-field term.

In the following we consider the limit of weakly coupled rung dimers, i.e. we set $J_{\perp}=1$ and consider $J_{\|} / J_{\perp} \equiv x$ as the small expansion parameter. The effective Hamiltonian $H_{\text {eff }}$ obtained by a quasiparticle-conserving CUT has the property $\left[H_{\text {eff }}, Q\right]=0$, meaning that the total number of triplons (dressed triplets which are the elementary excitations of the spin ladder) is a conserved quantity. The effective Hamiltonian at second order is

$$
H_{\mathrm{eff}}^{(2)}=Q+x T_{0}+\frac{x^{2}}{4}\left[T_{+2} T_{-2}-T_{-2} T_{2}\right]+H_{B} .
$$

The total spin $S_{\text {tot }}^{z}$ is a conserved quantity. The magnetic-field term is therefore not changed under the unitary transformation, and the low-energy physics is influenced solely by the local singlet $|s\rangle$ and the triplet $\left|t^{1}\right\rangle$ polarized parallel to the magnetic field (as discussed in Sec. 2). Identifying $|s\rangle$ with an empty site and $\left|t^{1}\right\rangle$ with the presence of a hard-core boson, one may deduce 
the effective Hamiltonian in this basis by calculating matrix elements on a finite cluster $[50,51]$. The result is

$$
\begin{aligned}
H_{\mathrm{eff}}^{h b}= & -t_{n} \sum_{i, j \in\{1,2\}}\left(b_{i}^{\dagger} b_{i+j}+\text { h.c. }\right)-\mu \sum_{i} n_{i} \\
& -t_{1}^{\prime} \sum_{i}\left(b_{i-1}^{\dagger} b_{i+1}+\text { h.c. }\right) n_{i}+v_{j} \sum_{i, j \in\{1,2\}} n_{i} n_{i+j},
\end{aligned}
$$

with $t_{1}=x / 2, t_{2}=x^{2} / 4, t_{1}^{\prime}=-x^{2} / 4, v_{1}=x / 2-3 x^{2} / 8, v_{2}=0$, and $\mu=B-1+3 x^{2} / 4$.

We emphasize that a calculation such as this, based on coupled dimers in an external magnetic field, can be performed for any frustrated lattice and to high orders in the perturbation. By using appropriate extrapolations, a quantitative low-energy effective Hamiltonian may be derived, which is usually applicable over a large part of the parameter space. The high-order expansion (including extrapolation of the bare series) becomes problematic only when the correlation length of the system exceeds the spatial range covered by the maximum order treated, for example close to a quantum phase transition. The method will break down if the ground state for the parameters of interest is not unitarily connected to the ground state about which one is expanding.

As a second example illustrating the importance of a quantitative effective model, meaning one obtained with high-order accuracy, we discuss the 2D spin-1/2 Heisenberg system known as the Shastry-Sutherland model [53] in a magnetic field,

$$
H=J^{\prime} \sum_{\langle i, j\rangle} S_{i} \cdot S_{j}+J \sum_{\ll i, j \gg} S_{i} \cdot S_{j}-B \sum_{i} S_{i}^{z} .
$$

The bonds denoted $\ll i, j \gg$ represent an array of orthogonal dimers, while the bonds $\langle i, j\rangle$, which are inter-dimer couplings, form a square lattice (Fig. 1.3). This theoretical model is believed to be realized experimentally in the layered copper oxide material $\mathrm{SrCu}_{2}\left(\mathrm{BO}_{3}\right)_{2}$, where the coupling ratio is $J^{\prime} / J \approx 0.63$. In the theoretical model, for $J^{\prime} / J$ smaller than a critical ratio of order 0.7 , the ground state of the model is given exactly by the product of dimer singlets, and the magnetization process of the system can be described in terms of hard-core bosons which, as discussed for the spin ladder above, represent polarized $\left|t^{1}\right\rangle$ triplons on the dimers, interacting and moving on an effective square lattice $[57,58]$.

A consequence of the strong frustration is the appearance of several magnetization plateaus which correspond to Mott-insulating phases of the hard-core bosons [54-56], where the translational symmetry of the system is broken and triplon excitations are frozen in the ground state as in a charge-ordered state (discussed in the chapter by Takigawa and Mila). Theoretically, all approaches to the basic theoretical model agree on the presence of magnetization plateaus at $1 / 3$ and $1 / 2$ of the saturation value [57-61], in agreement with experiments $[55,62]$. However, the structure below $1 / 3$ magnetization is rather 
controversial. On the experimental side, the original pulsed-field data show only two anomalies which were interpreted as plateaus at $1 / 8$ and $1 / 4$ [55], but the presence of additional phase transitions, and of a broken translational symmetry above the 1/8-plateau have been established by recent torque and NMR measurements performed up to $31 \mathrm{~T}[63,64]$. The possibility of additional plateaus has been pointed out by Sebastian et al. [62], who interpreted their high-field torque measurements as evidence for plateaus at $1 / q$, with $2 \leq q \leq 9$, and at $2 / 9$. On the theoretical side, the situation is also not settled. The finite clusters available for exact-diagonalization studies are not large enough to allow reliable predictions for high-commensurability plateaus, and the accuracy of the Chern-Simons mean-field approach initiated by Misguich et al. [60], and employed recently by Sebastian et al. [62] to explain their apparent additional plateaus, is not easy to assess. The essential difficulty lies in the fact that, because plateaus are a consequence of repulsive interactions between triplons, an accurate determination of the low-density, high-commensurability plateaus requires a precise knowledge of the long-range part of the interaction.

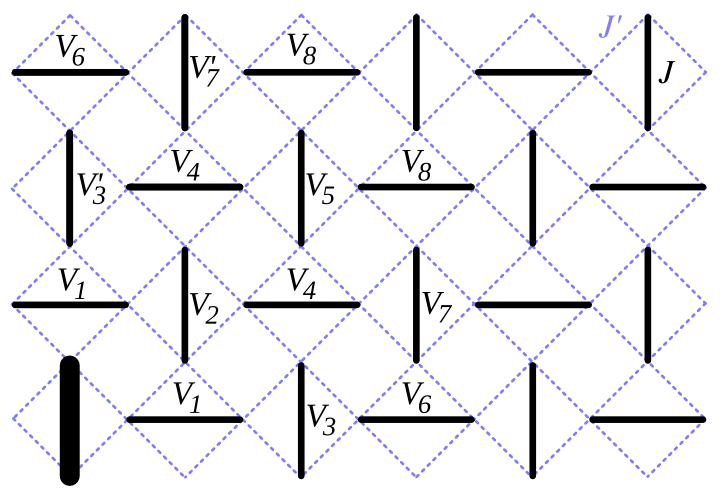

Fig. 1.3. Shastry-Sutherland lattice and definition of the two-body interactions. $V_{n}$ is the coefficient of the two-body interactions between the reference dimer (thick bond) and the corresponding dimer. Figure courtesy of Ref. [65].

Such a precise analysis was conducted recently using perturbative CUTs [65]. The processes relevant for the physics in a finite magnetic field are those with maximum total spin and total $S_{z}$. Thus the general form of the effective Hamiltonian obtained by the perturbative CUT takes the form

$$
H_{\mathrm{eff}}=\sum_{n=2,4,6 \ldots} \sum_{r_{1}, \ldots, r_{n}} C_{r_{1}, \ldots, r_{n}} b_{r_{1}}^{\dagger} \ldots b_{r_{n / 2}}^{\dagger} b_{r_{n / 2+1}} \ldots b_{r_{n}}
$$

where $r_{i}$ labels the sites of the square lattice formed by the $J$ bonds, while the hard-core boson operator $b_{r}^{\dagger}$ creates a polarized $\left|t^{1}\right\rangle$ triplon at site $r$. 
The coefficients $C_{r_{1}, r_{2}, \ldots, r_{n}}$ are then obtained as high-order series in $J^{\prime} / J$, computed up to order 15 for the two-body interactions $V_{n}$ to be discussed below.

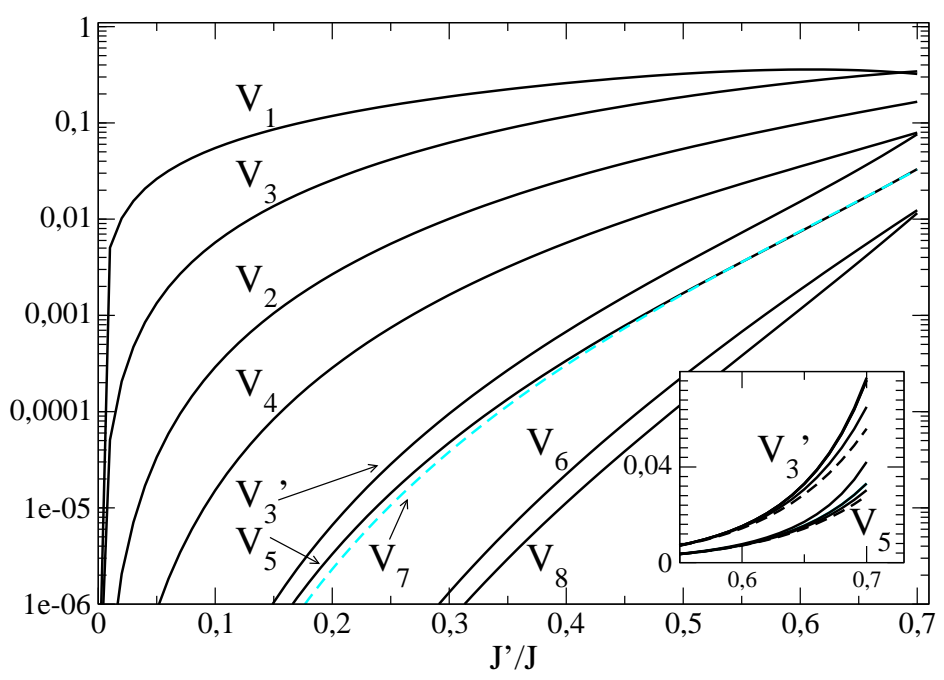

Fig. 1.4. Coefficients of the extrapolated two-body interactions $V_{n}$ as function of $J^{\prime} / J$. Inset: different extrapolations (solid lines) shown together with the bare series (dashed lines) for $V_{3}^{\prime}$ and $V_{5}$. Figure courtesy of Ref. [65].

It is found that the magnitudes of all the interaction terms decrease when the separation of the sites is increased. In addition, the physics at low density is dominated by the two-body density-density interactions, while the standard two-site hopping is, as expected, strongly suppressed due to the frustration [66]. The evolution with $J^{\prime} / J$ of the most relevant two-body interactions, defined in Fig. 1.3, is shown in Fig. 1.4. At small $J^{\prime} / J$, interactions beyond $V_{4}$ are small and may be neglected, but for larger coupling ratios the higher-order terms $V_{3}^{\prime}, V_{5}$, and $V_{7}$ (appearing at order 6 ) become important and contribute to the formation of low-density plateaus.

In general, the effective Hamiltonian $H_{\text {eff }}$ is by no means simpler than the original one, but it becomes so in the limit of low density and moderate $J^{\prime} / J$. Indeed, in this limit the kinetic terms are very small, and they can be considered as a perturbation of the interaction part, which is diagonal in the local Fock basis, $\left|n_{r_{1}}, n_{r_{2}}, \ldots\right\rangle$. It is thus appropriate to use a Hartree approximation in which the variational ground state is a product of local boson wave-functions, because this approximation becomes exact in the limit of vanishing kinetic energy. This approach has the further advantage that it can be used to compare and treat rather large unit cells, which is important in 
the $\mathrm{SrCu}_{2}\left(\mathrm{BO}_{3}\right)_{2}$ problem, where "solid" phases with a complicated structure can be found at low magnetizations.

The resulting phase diagram in the Hartree approximation is shown in Fig. 1.5. The phase diagram is dominated by a series of plateaus, which appear at $1 / 3$ and $1 / 2$ (not shown) even at very small $J^{\prime} / J$, and at $2 / 9,1 / 6,1 / 9$, and $2 / 15$ as $J^{\prime} / J$ is increased. A plateau structure of this kind is to be expected when the kinetic terms, as here, are very small, because if these terms were completely absent, the magnetization curve would consist simply of a sequence of plateaus. At $J^{\prime} / J=0.5$, the $1 / 6$-plateau is by far the most prominent stucture below $1 / 3$.

All of the plateaus found at low densities are actually stabilized by twobody repulsive interactions $V_{n}$ appearing at high orders in $J^{\prime} / J$. It is therefore crucial to obtain the effective Hamiltonian very accurately, because the most significant features in this density regime are found to result from the competition between small interactions.

Finally, it is worth adding that the method of CUTs is also able to treat observables $[36,50,65]$. To this end, an observable $O$ must be transformed by the same CUT as the Hamiltonian,

$$
\partial_{l} O(l)=[\eta(l), O(l)]
$$

yielding effective observables $O_{\text {eff }}=O(l=\infty)$. Here we mention only one possible application which can be very useful for the physics of frustrated quantum magnets: a typical situation in quantum magnetism is that, in addition to the dominant nearest-neighbor Heisenberg exchange interactions, there are small coupling terms $H_{\text {add }}$, such as Dzyloshinskii-Moriya interactions, which can have a profound influence on the physics of the system. Formally

$$
H_{\text {tot }}=H+H_{\text {add }},
$$

and it is both elegant and efficient to perform first a CUT on $H$, which contains the dominant couplings, and then to treat $H_{\text {add }}$ as an observable

$$
H_{\mathrm{tot}}^{\mathrm{eff}}=H^{\mathrm{eff}}+U^{\dagger} H_{\mathrm{add}} U .
$$

Here $H^{\text {eff }}$ conserves the number of quasiparticles, whereas the transformed observable $U^{\dagger} H_{\text {add }} U$ does not. Thus in the second step, performed after the first CUT, either ordinary perturbation theory or a second CUT can be applied to treat the term $U^{\dagger} H_{\text {add }} U$, which contains the small couplings. In cases where $H_{\text {add }}$ mixes low- and high-energy states, it is essential to retain access to the full Hilbert space of the problem.

\subsubsection{CORE (Contractor Renormalization)}

One further method which we highlight here for the derivation of low-energy effective models is the CORE technique, invented by Morningstar and Weinstein [68]. The underlying idea is to derive effective Hamiltonians for a truncated local basis in such a manner that the low-energy spectrum of the model 


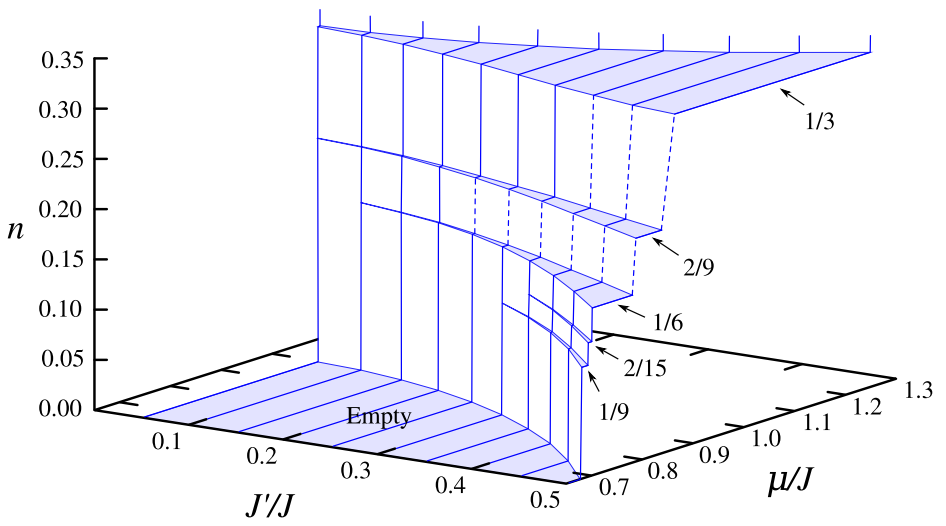

Fig. 1.5. Magnetization plateaus as a function of $\mu$ and $J^{\prime} / J$. The boson density $n$ is equal to the magnetization in units of the saturation value, and the chemical potential $\mu$ is equal to the magnetic field $B$. Solid lines denote results that are fully converged with respect to the terms retained in the Hamiltonian. Well-converged results are then connected by dashed lines. Figure courtesy of Ref. [65].

under study is reproduced exactly. As for CUTs, the CORE approach can be used either in an analytically oriented form [69-73] or as a numerical technique [74-77]. For recent reviews on CORE we refer the reader to Refs. [78,79].

The essential steps of the CORE algorithm are the following.

1) The system is divided into local subunits. One subunit is diagonalized, keeping $M$ suitable low-energy states.

2) The full Hamiltonian is diagonalized on a connected graph consisting of $N$ subunits. The low-energy eigenenergies $\epsilon_{n}$ and eigenstates $|n\rangle$ are calculated. 3) A basis of dimension $M^{N}$ is obtained by projecting the eigenstates onto the tensor product space of the retained states.

4) The effective Hamiltonian is constructed according to

$$
H_{N}^{\mathrm{eff}}=\sum_{n=1}^{M^{N}} \epsilon_{n}\left|\psi_{n}\right\rangle\left\langle\psi_{n}\right| .
$$

5) The connected range- $N$ interactions are determined by subtracting the contributions of all connected subclusters.

Finally, the effective Hamiltionian is deduced by a cluster expansion as

$$
H_{\mathrm{CORE}}^{\mathrm{eff}}=\sum_{i} H_{i}+\sum_{\langle i, j\rangle} H_{i j}+\ldots
$$

Note that $H_{\mathrm{CORE}}^{\mathrm{eff}}$ reproduces exactly the low-energy physics if one considers all of the terms on the right-hand side.

In practice, it is necessary to perform a truncation. The convergence of the algorithm therefore depends both on the range of the operators taken in the 
cluster expansion and on the number and type of low-energy states retained for one subunit. Hence the successful application of the CORE technique does require some physical insight concerning the problem at hand. However, once the relevant degrees of freedom have been selected, CORE represents a nonperturbative method for deriving effective low-energy Hamiltonians.

An important feature of the CORE algorithm is that it does not rely on the system being in a certain physical phase (to be contrasted with the example of quasiparticle-conserving CUTs discussed in the preceding section) and therefore does not break down even if a quantum phase transition takes place in the parameter space of the original model. As an example, we present here some illustrative CORE results for the magnetization curves of the $2 \mathrm{D}$ spin- $1 / 2$ Heisenberg model on the Shastry-Sutherland lattice discussed in the previous section [67]. The CORE technique is used to derive an effective Hamiltonian which is then treated by exact diagonalization (ED). The effective model obtained by CORE is found to agree very well with that obtained by perturbative CUTs. It is therefore expected that differences between the two approaches arise primarily from the method used to treat the effective model, which in the examples shown is either a classical limit [65] or ED [67].

Results obtained from CORE+ED are shown in Fig. 1.6. As in the treatment by a perturbative CUT, a rich plateau structure is found below $m=1 / 3$. This again highlights the utility of an approach to the physics by first deriving a quantitative effective model which is then treated by simpler techniques. One obvious advantage of ED compared to the classical treatment is that it takes quantum fluctuations fully into account. It is therefore striking that the magnetization curves in both approaches are dominated by the presence of plateaus. By contrast, one drawback in using ED as a solver for the effective

model (and one advantage of the classical solver) is the restriction on cluster sizes and shapes. Because the physics at low densities involves solid phases with rather large unit cells, it is a challenge within the ED approach to stabilize and to compare different solids, such as the 2/15 phase found in Ref. [65]. Despite the differing aspects, both positive and negative, of these approaches to this problem, it should be emphasized that the advanced techniques used to derive effective Hamiltonians have proven to be crucial in the discovery and resolution of the complicated magnetization processes of strongly frustrated quantum magnets.

\subsection{Conclusions}

In this chapter we have summarized a variety of tools which are used in the field of highly frustrated magnetism to derive effective low-energy Hamiltonians. We have aimed to capture the essential technical aspects of these different approaches and to provide examples of them for a number of physical applications. While it is not possible in a chapter of this type to cover all such 

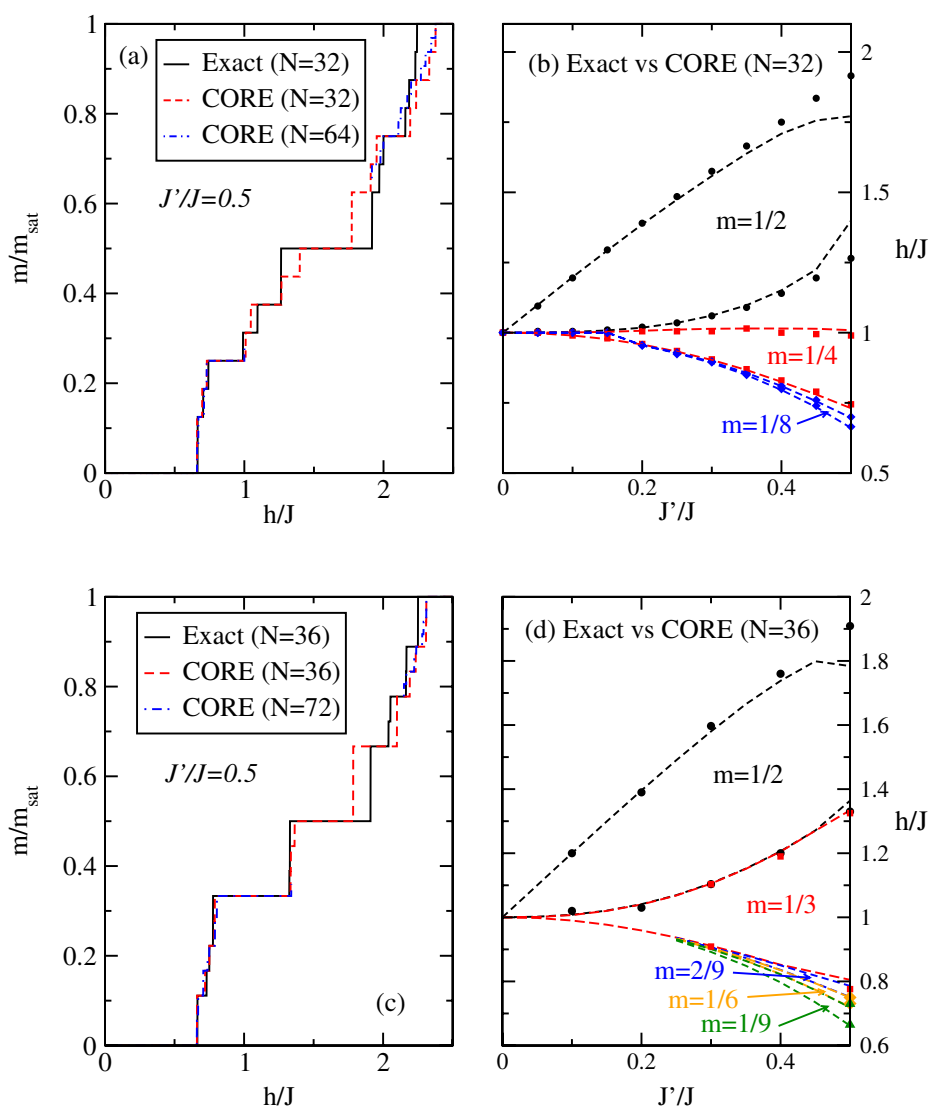

Fig. 1.6. (a) Magnetization curves obtained by ED and CORE calculations on an $N=32$ lattice and from CORE results with $N=64$ for $J^{\prime} / J=0.5$. (b) Phase diagram for $N=32$ as a function of $J^{\prime} / J$ and magnetic field $h / J$ : CORE results (lines) agree with $\mathrm{ED}$ (symbols) for locations of the $m=1 / 2,1 / 4$, and $1 / 8$ plateaus which are allowed on this cluster. (c,d) Same as (a,b) for $N=36$ and $N=72$. On these clusters, the $m=1 / 2,1 / 3,2 / 9,1 / 6$, and $1 / 9$ plateaus are allowed. Figure courtesy of Ref. [67].

methods in full detail, where relvant we have referred the interested reader to the more specialized literature.

We have shown that strong-coupling expansions and the derivation of effective models are at the same time standard techniques used by theoretical physicists for a broad range of physical questions and also a very active area of current research on strongly correlated quantum systems. For the first type of application, it is often the goal to identify the relevant low-energy degrees of freedom and to use an effective model to leading order in perturbation theory to understand qualitatively the phase diagram of a given model. By contrast, 
the aim of the current developments is to obtain a quantitative derivation of effective models and a complete understanding of the breakdown of such derivations.

\section{References}

1. See for example L. Landau and E. Lifshitz, Quantum mechanics, (Pergamon, 1991).

2. See for example P. Fulde, Electron Correlations in Molecules and Solids, (Springer, 1991).

3. T. Kato, Prog. Theor. Phys. 4, 514 (1949).

4. M. Takahashi, J. Phys. C 10, 1289 (1977).

5. P. W. Anderson, Phys. Rev. 115, 2 (1959).

6. K. Totsuka, Phys. Rev. B 57, 3454 (1998).

7. F. Mila, Eur. Phys. J. B 6, 201 (1998).

8. H. A. Bethe, Z. Phys. 71, 205 (1931).

9. A. Luther and I. Peschel, Phys. Rev. B 9, 2911 (1974).

10. F. D. M. Haldane, Phys. Rev. Lett. 45, 1358 (1980).

11. See for example D. C. Mattis, The theory of magnetism made simple, (World Scientific, 2006).

12. J.-B. Fouet, F. Mila, D. Clarke, H. Youk, O. Tchernyshyov, P. Fendley, and R. M. Noack, Phys. Rev. B 73, 214405 (2006).

13. R. Moessner, S. L. Sondhi, and P. Chandra, Phys. Rev. Lett. 84, 4457 (2000).

14. G. Misguich and F. Mila, Phys. Rev. B 77, 134421 (2008).

15. D. C. Cabra, M. D. Grynberg, P. C. Holdsworth, A. Honecker, P. Pujol, J. Richter, D. Schmalfuss, and J. Schulenburg, Phys. Rev. B 71, 144420 (2005).

16. D. L. Bergman, R. Shindou, G. A. Fiete, and L. Balents, Phys. Rev. B 75, 094403 (2007).

17. I. Rousochatzakis, A. M. Läuchli, and F. Mila, Phys. Rev. B 77, 094420 (2008).

18. H. J. Schulz in Proceedings of the XXXIst Rencontres de Moriond, edited by T. Martin, G. Montambaux, and J. Trin Thanh Vin (Editions Frontières, Gifsur-Yvette, 1996).

19. V. Subrahmanyam, Phys. Rev. B 52, 1133 (1995).

20. F. Mila, Phys. Rev. Lett. 81, 2356 (1998).

21. For a detailed discussion of this point, see M. Ferrero, F. Becca, and F. Mila, Phys. Rev. B 68, 214431 (2003).

22. P. Lecheminant, B. Bernu, C. Lhuillier, L. Pierre, and P. Sindzingre, Phys. Rev. B 56, 2521 (1997).

23. B. Damski, H.-U. Everts, A. Honecker, H. Fehrmann, L. Santos, and M. Lewenstein, Phys. Rev. Lett. 95, 060403 (2005).

24. H. Tsunetsugu, Phys. Rev. B 65, 024415 (2002).

25. V. N. Kotov, M. E. Zhitomirsky, M. Elhajal, and F. Mila, Phys. Rev. B 70, 214401 (2004); V. N. Kotov, M. Elhajal, M. E. Zhitomirsky, and F. Mila, Phys. Rev. B 72, 014421 (2005).

26. H. J. Schmidt and Y. Kuramoto, Physica C 167, 263 (1990).

27. M. Takahashi, J. Phys. C: Solid State Phys. 10, 1289 (1977).

28. A. H. MacDonald, S. M. Girvin, and D. Yoshioka, Phys. Rev. B 41, 2565 (1990).

29. E. Müller-Hartmann and A. Reischl, Eur. Phys. J. B 28, 173 (2002). 
30. F. J. Wegner, Ann. Physik 3, 77 (1994).

31. S. D. Głazek and K. G. Wilson, Phys. Rev. D 48, 5863 (1993).

32. S. D. Głazek and K. G. Wilson, Phys. Rev. D 49, 4214 (1994).

33. C. Knetter, K. P. Schmidt, M. Grüninger, and G. S. Uhrig, Phys. Rev. Lett. 87, 167204 (2001).

34. P. Lenz and F. Wegner, Nucl. Phys B 482, 693 (1996).

35. K. G. Wilson, Rev. Mod. Phys. 47, 773 (1975).

36. S. Kehrein, Flow-equation approach to many-particle systems, Springer Tracts in Modern Physics 217 (2006), and references therein.

37. J. Stein, J. Stat. Phys. 88, 487 (1997).

38. A. Mielke, Eur. Phys. J. B 5, 605 (1998).

39. G. S. Uhrig and B. Normand, Phys. Rev. B 58, R14705 (1998).

40. C. Knetter and G. S. Uhrig, Eur. Phys. J. B 13, 209 (2000).

41. C. Knetter, A. Bühler, E. Müller-Hartmann, and G. S. Uhrig, Phys. Rev. Lett. 85, 3958 (2000).

42. C. P. Heidbrink and G. S. Uhrig, Eur. Phys. J. B 30, 443 (2002).

43. W. Brenig, Phys. Rev. B 67, 064402 (2003).

44. K. P. Schmidt and G. S. Uhrig, Mod. Phys. Lett. B 19, 1179 (2005).

45. A. Reischl, E. Müller-Hartmann, and G. S. Uhrig, Phys. Rev. B 70, 245124 (2004).

46. S. Dusuel and G. S. Uhrig, J. Phys. A: Math. and Gen. 37, 9275 (2004).

47. S. Dusuel and J. Vidal, Phys. Rev. Lett. 93, 237204 (2004).

48. J. N. Kriel, A. Y. Morozov, and F. G. Scholtz, J. Phys. A: Math. and Gen. 38, 205 (2005).

49. K. P. Schmidt, S. Dusuel, and J. Vidal, Phys. Rev. Lett. 100, 057208 (2008).

50. C. Knetter, K. P. Schmidt, and G. S. Uhrig, J. Phys.: Condens. Matter 36, 7889 (2003).

51. C. Knetter, K. P. Schmidt, and G. S. Uhrig, Eur. Phys. J. B 36, 525 (2004).

52. S. Sachdev and R. N. Bhatt, Phys. Rev. B 41, 9323 (1990).

53. B. S. Shastry and B. Sutherland, Physica B 108B, 1069 (1981).

54. E. Müller-Hartmann, R. R. P. Singh, C. Knetter, and G. S. Uhrig, Phys. Rev. Lett. 81, 1808 (2000).

55. K. Onizuka et al., J. Phys. Soc. Jpn. 69, 1016 (2000).

56. K. Kodama et al., Science 298, 395 (2002).

57. T. Momoi and K. Totsuka, Phys. Rev. B 62, 15067 (2000).

58. S. Miyahara and K. Ueda, J. Phys.: Condens. Matter 15, R327 (2003).

59. S. Miyahara and K. Ueda, Phys. Rev. B 61, 3417 (2000).

60. G. Misguich, T. Jolicoeur, and S. M. Girvin, Phys. Rev. Lett. 87, 097203 (2001).

61. S. Miyahara, F. Becca and F. Mila, Phys. Rev. B 68, 024401 (2003).

62. S. E. Sebastian, N. Harrison, P. Sengupta, C. D. Batista, S. Francoual, E. Palm, T. Murphy, H. A. Dabkowska, and B. D. Gaulin, Proc. Nat. Acad. Sci. 105, 20157 (2008).

63. F. Levy, I. Sheikin, C. Berthier, M. Horvatić, M. Takigawa, H. Kageyama, T. Waki, and Y. Ueda, Europhys. Lett. 81, 67004 (2008).

64. M. Takigawa, S. Matsubara, M. Horvatić, C. Berthier, H. Kageyama, and Y. Ueda, Phys. Rev. Lett. 101, 037202 (2008).

65. J. Dorier, K. P. Schmidt, and F. Mila, Phys. Rev. Lett. 101, 250402 (2008).

66. S. Miyahara and K. Ueda, Phys. Rev. Lett. 82, 3701 (1999).

67. A. Abendschein and S. Capponi, Phys. Rev. Lett. 101, 227201 (2008). 
68. C. J. Morningstar and M. Weinstein, Phys. Rev. Lett. 73, 1873 (1994); Phys. Rev. D 54, 4131 (1996).

69. M. Weinstein, Phys. Rev. B 63, 174421 (2001).

70. E. Altman and A. Auerbach, Phys. Rev. B 65, 104508 (2002).

71. E. Berg, E. Altmann, and A. Auerbach, Phys. Rev. Lett. 90, 147204 (2003).

72. R. Budnik and A. Auerbach, Phys. Rev. Lett. 93, 187205 (2004).

73. M. S. Siu and M. Weinstein, Phys. Rev. B 75, 184403 (2007).

74. J. Piekarewicz and J. R. Shepard, Phys. Rev. B 56, 5366 (1997); Phys. Rev. B 57, 10260 (1998).

75. S. Capponi and D. Poilblanc, Phys. Rev. B 66, 180503(R) (2002).

76. S. Capponi, A. Läuchli, and M. Mambrini, Phys. Rev. B 70, 104424 (2004).

77. A. Abendschein and S. Capponi, Phys. Rev. B 76, 064413 (2007).

78. A. Auerbach, AIP Conf. Proc. 8161 (2006).

79. S. Capponi AIP Conf. Proc. 81616 (2006). 